\section{Lumbo-sacral spine surgery and severe bradycardia}

To the Editor:

A 37-yr-old woman weighing $95 \mathrm{~kg}$ underwent L5 to S1 spinal decompression for left sided lower leg pain and weakness. Preoperative evaluation revealed no history of heart disease, arrhythmia or syncope, and no baseline electrocardiogram (ECG) was ordered for this otherwise healthy patient. Her only medication was naproxen $500 \mathrm{mg}$ po $\mathrm{q} 12 \mathrm{hr}$ prn. After an uneventful induction with $35 \mu \mathrm{g}$ of sufentanil, $150 \mathrm{mg}$ of propofol and $50 \mathrm{mg}$ of rocuronium, endotracheal intubation was performed and general anesthesia was maintained with desflurane and a sufentanil infusion of $5 \mu \mathrm{g} \cdot \mathrm{hr}^{-1}$. The patient was then placed in the prone position. Her vital signs were all within normal range when the incision was made. As the surgery progressed, two episodes of relative bradycardia, heart rate $(\mathrm{HR})<45$ beats. $\mathrm{min}^{-1}$ were observed which occurred coincidentally with the surgeons brief use of the electrocautery in the lumbo-sacral segments. Both times, the patient's HR quickly increased to a baseline of 85 beats. $\mathrm{min}^{-1}$ when stimulation was stopped, and the episodes were wrongly interpreted as interference with the ECG. Shortly thereafter, as the surgeons were maintaining hemostasis with the prolonged use of the electrocautery, HR decreased below 10 beats. $\mathrm{min}^{-1}$ for at least 30 to $35 \mathrm{sec}$. Atropine $(0.6 \mathrm{mg})$ was given twice, which increased HR to 40 beats. $\mathrm{min}^{-1}$. Ephedrine $(10 \mathrm{mg})$ was then given and HR rose to 100 beats $\mathrm{min}^{-1}$, to slowly stabilize around 75 beats. $\mathrm{min}^{-1}$ for the remainder of the surgery. There was no non-invasive blood pressure measurement during the period of severe bradycardia. For the first ten minutes post-ephedrine, the patient was hypertensive $(180 / 100$ at five minutes and $160 / 90 \mathrm{mmHg}$ at ten minutes). All other blood pressures were within normal limits. A Medline search from 1966 to 2003 did not disclose similar occurrences of severe bradycardia during lumbo-sacral spine surgery. We interpret the episode of severe bradycardia in this otherwise healthy patient as a stimulation of afferent parasympathetic nerve endings causing a reflex-celiac (vagovagal) reaction. This reaction would be a parallel to reflex bradycardia seen with rectal distention or testicular manipulation. No untoward event resulted from this episode and the patient recovered normally from the surgery and anesthesia. We therefore suggest vigilance with surgery in the region of the posterior sacral segments. This episode reinforces our belief in the practice of having vagolitic and sympathomimetic drugs available at all times to rapidly treat hemodynamically compromising episodes of severe bradycardia.

Alain Deschamps PhD MD FRCPC
Montréal, Québec

\section{Ultrasound imaging in pediatric regional anesthesia}

To the Editor:

Real-time ultrasound (US) imaging can facilitate the performance of both peripheral or plexus blocks and neuraxial blocks ${ }^{1}$ in adults. Although US has been used as a screening tool for spinal anomalies in children, ${ }^{2}$ it has not been investigated in pediatric regional anesthesia.

Pediatric patients require sedation or anesthesia for regional blocks. ${ }^{3}$ This makes regional anesthesia more challenging and increases the risk. ${ }^{4}$

After approval of the local Ethics Committee and written informed consent, we evaluated US in infants. The US images were acquired under general anesthesia. We present the example of a six-month-old child scheduled for surgical reconstruction of anal atresia; the colostomy had been performed at ten days of age. For intra- and postoperative pain relief caudal block was used first and an epidural catheter was placed for the second procedure using US imaging.

A Logic 400 CL US machine (General Electric, Solingen, Germany) with a linear 7.5 MHz US probe was used. With the patient in a right lateral decubitus position US scanning was performed in longitudinal and transverse planes at sacral (S2), lumbar (L3-4), and thoracic (T6-7) levels at the age of one week and six months.

The intraspinal and extraspinal anatomy can be recognized easily (Figure). US imaging enables an exact representation of the epidural space and of the intrathecal structures, the dural sac and the cauda equina, with a high resolution. The nerve roots of the cauda equina are seen as a hyperechogenic signals and the longitudinal nerve roots are displayed as single fibres. The dura mater is defined by a typical double-layer hyperechogenic signal. Imaging at six months clearly demonstrates the cephalad regression of the dural sac. Increased calcification of the vertebral column causes a reduction of the available acoustic window.

The quality of real-time images via an acoustic window through the skeletal framework shows that US is useful for imaging neuraxial structures relevant to pediatric neuraxial anesthesia. The exact depth to the dura 


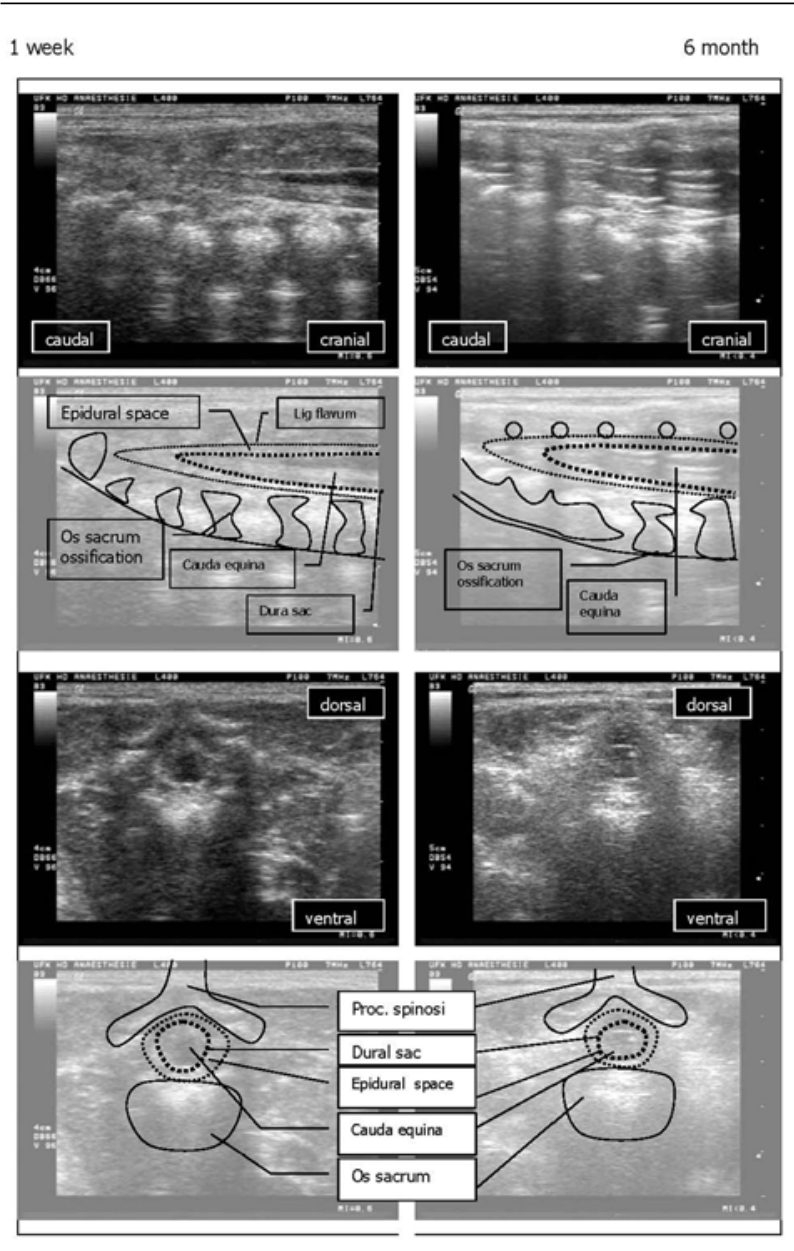

FIGURE Lumbar and sacral areas imaged at one week and six months.

mater can be predetermined before needle advancement. ${ }^{5}$ Screening for congenital anatomical variations is possible and structures identified through this acoustic window enhance efficacy and safety. The quality of the images acquired in infants are superior to those in adults, possibly because of the smaller depth and poorly ossified structures. The use of real-time imaging can facilitate needle and catheter placement.

In conclusion, US imaging can make pediatric regional anesthesia easier and more secure. Important anatomical details relevant to neuraxial anesthesia can be visualized. In addition, it is a useful tool for teaching the principles of pediatric neuraxial regional anesthesia.

Hans-Jürgen Rapp MD

Thomas Grau MD

Heidelberg, Germany

\section{References}

1 Peterson MK, Millar FA, Sheppard DG. Ultrasoundguided nerve blocks. Br J Anaesth 2002; 88: 621-4.

2 Dick EA, Patel K, Owens CM, De Bruyn R. Spinal ultrasound in infants. Br J Radiol 2002; 75: 384-92.

3 Coley BD, Shiels WE 2nd, Hogan MJ. Diagnostic and interventional ultrasonography in neonatal and infant lumbar puncture. Prediatr Radiol 2001; 31: 399-402.

4 Rose JB. Spinal cord injury in a child after single-shot epidural anesthesia. Anesth Analg 2003; 96: 3-6.

5 Gran T, Leipold RW, Conradi R, Martin E, Motsch J. Efficacy of ultrasound imaging in obstetric epidural anesthesia. J Clin Anesth 2002; 14: 169-75.

\section{The "bouncing sign" may optimize the insertion of the laryngeal tube}

To the Editor:

The laryngeal tube (LT; VBM, Medizintechnik, GmbH, Germany) is a new, FDA approved, supraglottic airway device. The LT is inserted blindly along the hard palate and hypopharynx into the esophagus until the centre black line on the tube is level with the teeth and the tip lies behind the cricoid (C6 level). Two cuffs - one distal, esophageal and one proximal, pharyngeal isolate the glottis for ventilation. ${ }^{1,2}$ Malposition is usually defined after inflation of the cuffs (proximal blue cuff easily visible in the mouth, low leak pressures, gastric insufflation, and failed ventilation).

One of the characteristics of the LT is the short tube length; subsequently the recommended depth may be achieved with the distal-esophageal-tip embedded in the glottic/periglottic elastic structures (e.g., the piriform sinus, a down folded epiglottis...). When released, a malpositioned LT will "bounce back" from the intended position pushed by the elastic recoil of the aforementioned structures ("positive bouncing sign"). In a succession of 75 adult male patients we observed the "bouncing sign" in five cases. Malposition was confirmed after inflation. The bouncing was variable $(\mathrm{mm}$ to $\mathrm{cm}$ ) but with the same significance. In the 70 patients with "negative bouncing sign" the distal tip of the device was inserted in the esophagus and correct positioning was confirmed clinically after inflation of the cuffs: bilateral breath sounds with easy ventilation, peak airway pressure $>20 \mathrm{~cm} \mathrm{H}_{2} \mathrm{O}$, no gastric insufflation, proximal blue cuff minimally visible in the oropharynx and a square wave capnographic trace.

Our current approach is to reposition an LT that "bounces back" without completely removing it from the oral cavity. Adjunct maneuvers (jaw lift, jaw 